\title{
Spontaneous deep vein thrombosis in the upper extremity of a 45-year-old woman
}

\author{
Corey M. Stein MD, Anne McLeod MD, Luke A. Devine MD MHPE
}

See also www.cmaj.ca/lookup/doi/10.1503/cmaj.140552

Competing interests: Anne McLeod has received consultancy fees from

Leo Pharma Inc. to provide education on the use of low-molecular-weight heparin. No other competing interests were declared.

This article has been peer reviewed.

The authors have obtained patient consent.

Correspondence to: Luke Devine, ldevine@mtsinai.on.ca

CMAJ 2015. DOI:10.1503 /cmaj.140400
1 45-year-old woman presented to her family doctor following a three-day history of acute-onset, progressive swelling and sensation of heaviness in her left upper extremity. In addition, she described pleuritic chest pain and dyspnea on exertion. Five days before presentation, she had been lifting heavy furniture and boxes and had performed her regular exercise routine that involved lifting 10 - and 15 -pound weights overhead. She had no history of thrombosis and no family history of clotting disorders. Her medical history included Hashimoto thyroiditis and remote iron deficiency anemia secondary to menorrhagia. Cancer screening had yielded normal results on a Papanicolaou test and a colonoscopy performed three years earlier. She had no recent immobilization, surgery or long-distance travel and was not taking supplemental estrogen. The patient was referred to the emergency department for evaluation of suspected deep vein thrombosis (DVT) in her left upper extremity and pulmonary embolus.

In the emergency department, she had a regular heart rate of 96 beats/min and an oxygen saturation of $95 \%$ on room air. Her temperature and blood pressure were within normal limits. There was obvious swelling of the entire left

\section{- Ker Points}

- Paget-Schroetter syndrome refers to spontaneous thrombosis (unrelated to cancer, thrombophilia or an indwelling central venous catheter) of the subclavian vein at the thoracic outlet due to extrinsic venous compression that is accentuated by exertion of the upper extremity.

- The syndrome should be considered in any young, otherwise healthy individual who presents with unexplained acute-onset unilateral swelling of an upper extremity.

- Treatment is aimed at preventing pulmonary embolism, recurrent thrombosis and post-thrombotic syndrome.

- Strong evidence to guide treatment is lacking; treatment options range from anticoagulation alone to catheter-directed thrombolysis and surgical decompression of the thoracic outlet. upper extremity, dilated subcutaneous collateral veins over the anterior shoulder, and mild cyanosis in the hand and fingers. Neurologic and arterial examinations of the upper extremities yielded normal findings. Venous duplex ultrasonography showed an occlusive thrombus in the left subclavian and axillary veins. Computed tomography pulmonary angiography showed pulmonary emboli in the bilateral subsegmental arteries. The patient was admitted, and treatment with low-molecular-weight heparin was started.

The following morning, the patient underwent peripheral venography (Figure 1) with catheter-directed thrombolysis using a tissue plasminogen activator. She subsequently received ongoing anticoagulation with intravenous unfractionated heparin. A follow-up venogram 24 hours after the thrombolysis showed resolution of the clot; however, severe stenosis of the left subclavian vein was noted. Angioplasty of the stenotic area was only partially successful, leaving residual stenosis of the subclavian vein in the neutral position, with dynamic occlusion on abduction of the left upper extremity (Figure 2). Two days later, the patient was discharged home and prescribed low-molecularweight heparin.

Two months after discharge, the patient underwent an uncomplicated transaxillary resection of the left first rib to relieve compression of the subclavian vein. Venography performed one month later showed resolution of the subclavian vein stenosis and of the dynamic occlusion (Figure 3). In discussion with her treating physician, the patient elected to complete a further three months of anticoagulation with therapeutic doses of low-molecular-weight heparin instead of transitioning to oral anticoagulation following her surgery. She experienced good functional recovery of her left upper extremity, with no recurrence of DVT. 


\section{Discussion}

Paget-Schroetter syndrome, also known as effort thrombosis, refers to spontaneous thrombosis of the subclavian vein at the thoracic outlet due to extrinsic venous compression. ${ }^{1}$ It is clinically distinct from thrombosis occurring secondary to cancer, thrombophilia or central venous catheterization. Paget-Schroetter syndrome occurs as a result of an anatomic abnormality (congenital or acquired) at the costoclavicular junction, which leads to compression of the subclavian vein. ${ }^{1}$ Compression is accentuated by exertion of the upper extremity and results in microtrauma of the endothelium, with subsequent activation of the coagulation cascade, venous intimal hyperplasia and fibrosis. Acute thrombosis can result, which is often superimposed on chronic venous stenosis. $^{2}$

Paget-Schroetter syndrome is relatively rare, with an annual incidence of 1-2 cases per 100000 population. ${ }^{2}$ Nevertheless, it is important to recognize the clinical presentation and understand management options to optimize patient outcomes. As will be discussed later, treatment with anticoagulation alone will not relieve the intermittent obstruction, and therefore there is a risk of persistent symptoms and recurrence of thrombosis. ${ }^{3}$ Patients who are referred to vascular centres may be offered catheter-directed thrombolysis followed by consideration for surgical decompression of the thoracic outlet. Although no randomized trials of anticoagulation versus surgical management exist, some observational studies suggest that establishment of venous patency and definitive management of the obstruction may lead to better functional outcomes. ${ }^{1}$

\section{Clinical presentation}

The classic presentation of Paget-Schroetter syndrome includes the abrupt onset of swelling of an entire upper extremity, accompanied by pain and a sensation of heaviness in the limb. Patients are typically 15-45 years of age and are frequently physically active, with many involved in activities that require heavy lifting or repetitive overhead movements. ${ }^{1,4}$ As was consistent with our patient, about two-thirds of patients will recall a history of strenuous use of the upper extremity before the onset of symptoms. ${ }^{5}$ Some patients who experience intermittent obstruction of venous outflow or recurrent partial thrombosis may notice the intermittent occurrence of similar, but less severe symptoms before the acute symptoms of Paget-Schroetter syndrome develop. ${ }^{2}$

Physical examination reveals edema of the affected upper extremity, which can be associ- ated with distal cyanosis of the hands and fingers. Dilated subcutaneous collateral veins over the anterior shoulder may also be seen. Although more common with DVT in the lower extremities, pulmonary embolism occurs in 10\%-20\% of people with DVT in the upper extremities. ${ }^{4}$

\section{Diagnosis}

Paget-Schroetter syndrome should be considered in any young, otherwise healthy individual who presents with unexplained acute-onset swelling of an upper extremity. Venous compression ultrasonography is the diagnostic test of choice for DVT in the upper extremities, because it has high sensitivity (90\%-100\%) and specificity (87\%$100 \%) .{ }^{5}$ However, if a thrombus is restricted to the proximal subclavian or brachiocephalic vein, which can be obscured by surrounding bony structures, compression ultrasonography may yield a false-negative result. Computed tomography and magnetic resonance venography may provide additional anatomic information; however, the performance characteristics of these modalities are not well established for the diagnosis of upper-extremity DVT.

Although ultrasonography is the initial diagnostic test of choice, venography has traditionally been the reference standard for the diagnosis of Paget-Schroetter syndrome. It is generally

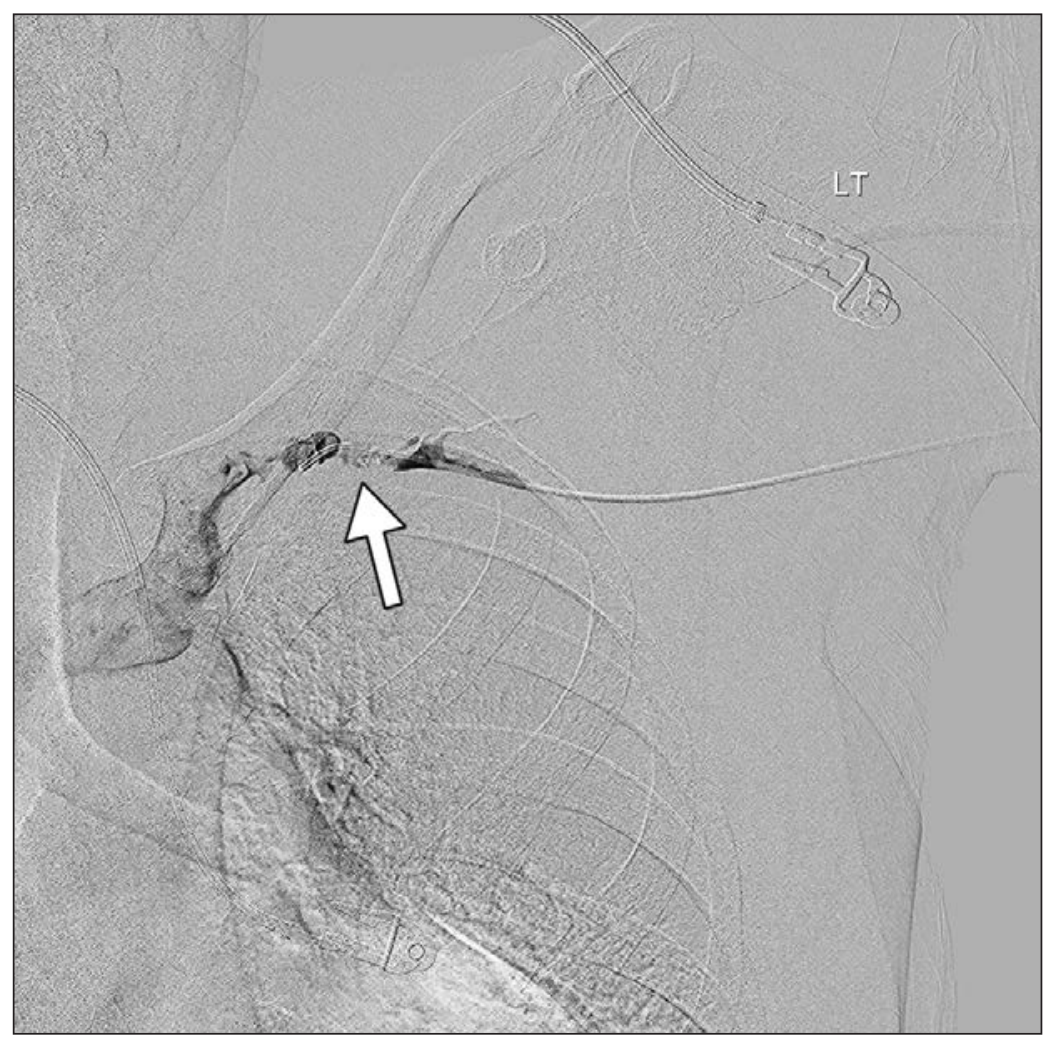

Figure 1: Venogram in a 45-year-old woman with acute-onset swelling of her left upper extremity, showing deep vein thrombosis involving the subclavian vein (arrow), with extension to the axillary and brachiocephalic veins. 
performed only if thrombolysis is planned or the diagnosis is uncertain. ${ }^{5}$ In addition to identifying the thrombus, venography provides visualization of the collateral circulation, can identify areas of stenosis of the subclavian vein and enables administration of thrombolytics for definitive treatment of an identified thrombus. ${ }^{2}$

Patients with a history of thrombosis in another vessel or a family history of thrombosis warrant further consideration for screening for thrombophilia. Screening is not routinely recommended in patients with Paget-Schroetter syndrome. Observational studies have shown that coagulation abnormalities are less likely to be found in patients with the syndrome than in

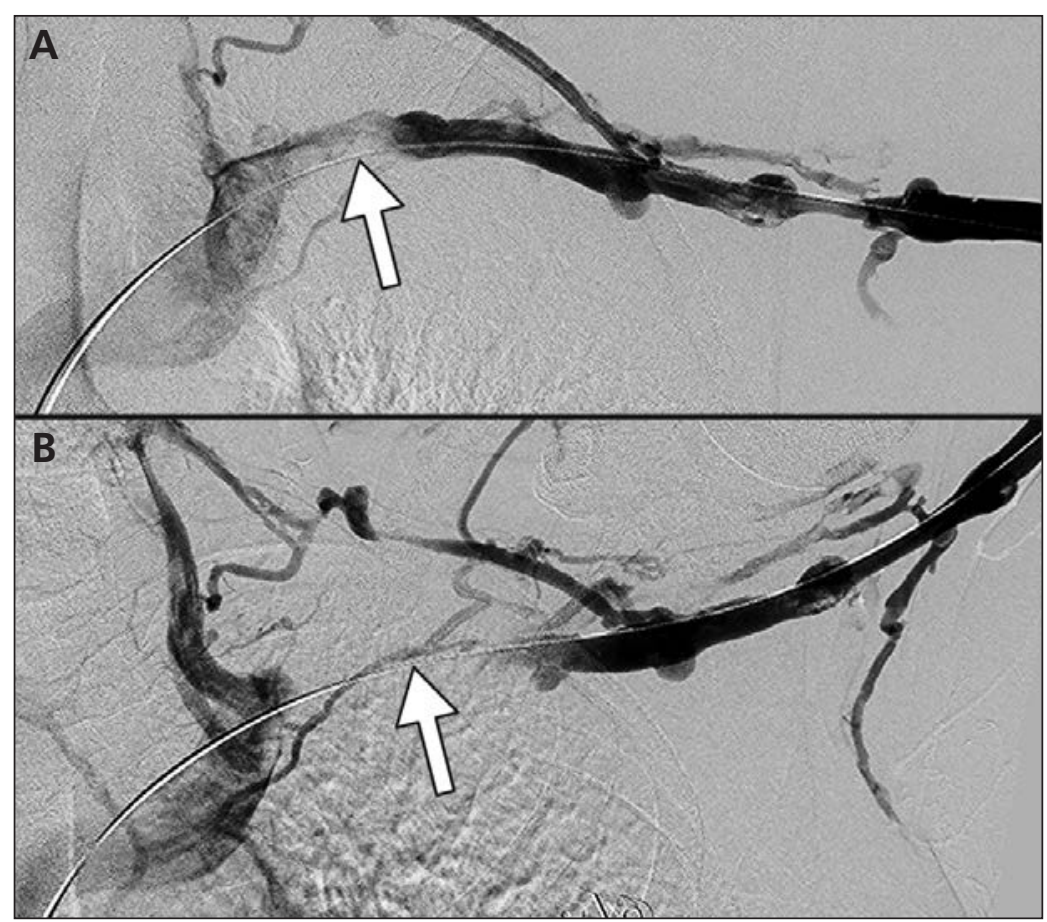

Figure 2: Venograms after thrombolysis and angioplasty of the left upper extremity, showing (A) mild stenosis of the subclavian vein (arrow) in the neutral position and (B) complete occlusion of the vein (arrow) from compression at the costaclavicular junction with the upper extremity abducted to 120 degrees.

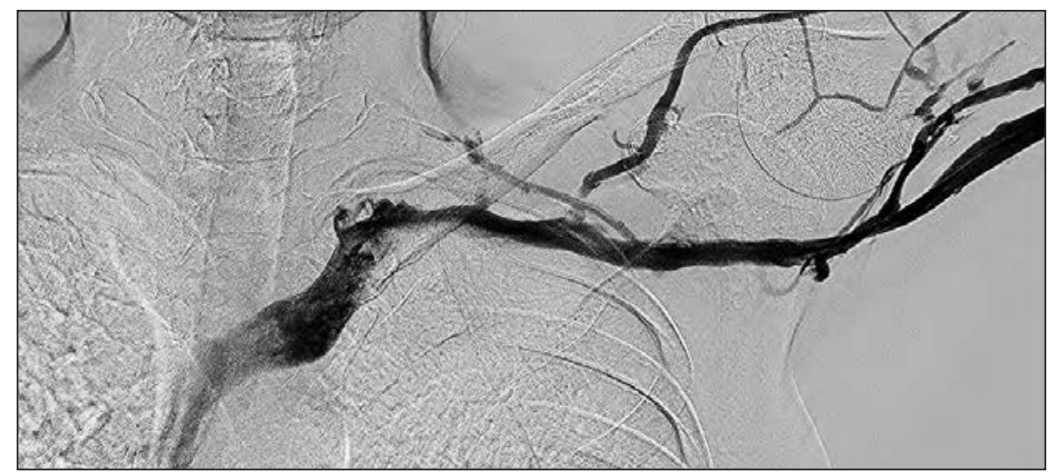

Figure 3: Venogram 1 month after resection of the first rib on the left side, showing a widely patent subclavian vein with the upper extremity abducted to 120 degrees. those with DVT in a lower extremity and that the incidence among patients with the syndrome may be similar to that in the general population. ${ }^{6}$

\section{Management}

Given the relative rarity of the condition and the lack of randomized controlled trials, the optimal management of Paget-Schroetter syndrome has not been established. For many years, treatment with anticoagulation alone was the standard of care, but subsequent long-term data have shown a high incidence of post-thrombotic syndrome, a potentially disabling condition characterized by chronic arm swelling and pain. Small cohort studies involving patients with Paget-Schroetter syndrome given anticoagulation alone showed that post-thrombotic syndrome occurred in $27 \%$ $46 \%$ of patients. ${ }^{3,7,8}$

As a result of the disappointing results achieved with anticoagulation alone, the use of thrombolysis has become more widespread in the hope that early restoration of venous patency will reduce the incidence of post-thrombotic syndrome. Thrombolysis is performed in experienced centres in patients with a low risk of bleeding and severe symptoms that are consistent with Paget-Schroetter syndrome. ${ }^{5}$ Catheter-directed thrombolysis has traditionally been used, because it achieves higher rates of clot resolution and a lower incidence of bleeding compared with systemic thrombolysis. ${ }^{5}$ Thrombolysis is most successful when administered early. In one case series of 26 patients, successful lysis occurred in $73 \%$ of those in whom thrombolysis was performed within 14 days of symptom onset and in none when performed after 14 days. $^{9}$

The American College of Chest Physicians guideline suggests anticoagulant therapy over thrombolysis (weak recommendation based on low- or very-low-quality evidence), although it does not distinguish between Paget-Schroetter syndrome and other causes of upper-extremity DVT. ${ }^{10}$ Furthermore, the guideline acknowledges that thrombolysis should be considered in patients with good functional status who present with severe symptoms. Patients who choose thrombolysis attach a higher value to preventing post-thrombotic syndrome and are more willing to accept the increased initial risk of bleeding. ${ }^{10}$

Among patients who undergo successful thrombolysis, clots will recur in $25 \%-34 \%$ if the underlying anatomic problem is not corrected. ${ }^{2,11}$ Angioplasty and stenting of the subclavian vein have been attempted but generally fail at high rates. ${ }^{2}$ Although some experts feel that decompression of the thoracic outlet is required, usually through transaxillary resection of the first rib, controversy remains regarding the need for 
surgery in all patients. Some centres perform surgery only in patients who have persistent symptoms or recurrence of thrombosis and have found that $43 \%$ of patients avoided surgery with this approach. ${ }^{11}$ Others operate on all patients. In one retrospective case series of 506 patients who received thrombolysis and transaxillary resection of the first rib, 486 (96\%) experienced good to excellent long-term clinical results after a mean follow-up period of 7.2 years. ${ }^{1}$

The optimal timing of surgery is also debated. Historically, surgery was performed three months after thrombolysis to allow local inflammation to resolve. This delay, however, was associated with a recurrence of DVT in $10 \%$ or more of patients waiting for surgery. ${ }^{2}$ Early surgery, within three days of thrombolysis, has been shown in observational studies to be successful and to have a risk of bleeding complications similar to that associated with delayed surgery. ${ }^{2}$

Based on findings from prospective observational studies and extrapolation from the literature on lower-extremity DVT, the American College of Chest Physicians guideline recommends that patients with acute upper-extremity DVT receive three months of therapeutic anticoagulation, regardless of the treatment approach chosen. ${ }^{10}$

\section{References}

1. Urschel HC, Patel AN. Surgery remains the most effective treatment for Paget-Schroetter syndrome: 50 years' experience. Ann Thorac Surg 2008;86:254-60.

2. Illig KA, Doly AJ. A comprehensive review of Paget-Schroetter syndrome. J Vasc Surg 2010;51:1538-47.

3. Persson LM, Arnhjort T, Larfars G, et al. Hemodynamic and morphologic evaluation of sequelae of primary upper extremity deep vein thromboses treated with anticoagulation. $J$ Vasc Surg 2006;43:1230-5.

4. Thompson RW. Comprehensive management of subclavian vein effort thrombosis. Semin Intervent Radiol 2012;29:44-51.

5. Kucher N. Clinical practice. Deep-vein thrombosis of the upper extremities. N Engl J Med 2011;364:861-9.

6. Héron E, Lozinguez O, Alhenc-Gelas M, et al. Hypercoagulable states in primary upper-extremity deep vein thrombosis. Arch Intern Med 2000;160:382-6.

7. Prandoni P, Bernardi E, Marchiori A, et al. The long term clinical course of acute deep vein thrombosis of the arm: prospective cohort study. BMJ 2004;329:484-5.

8. Kahn SR, Elman EA, Bornais C, et al. Post-thrombotic syndrome, functional disability and quality of life after upper extremity deep venous thrombosis in adults. Thromb Haemost 2005;93:499-502.

9. Doyle A, Wolford HY, Davies MG, et al. Management of effort thrombosis of the subclavian vein: today's treatment. Ann Vasc Surg 2007;21:723-9.

10. Kearon C, Aki EA, Comerota AJ, et al. Antithrombotic therapy for VTE disease: antithrombotic therapy and prevention of thrombosis, 9th ed: American College of Chest Physicians evidencebased clinical practice guidelines. Chest 2012;141(2 Suppl): e419S-94S.

11. Lee JT, Karwowski JK, Harris EJ, et al. Long-term thrombotic recurrence after nonoperative management of Paget-Schroetter syndrome. J Vasc Surg 2006;43:1236-43.

Affiliations: Department of Medicine (Stein, McLeod, Devine), University of Toronto; Division of General Internal Medicine (Devine), Mount Sinai Hospital; Division of Medical Oncology and Hematology (McLeod), Sunnybrook Hospital, Toronto, Ont.

Contributors: All of the authors made substantial contributions to this work. Luke Devine and Corey Stein contributed to the design and conception of the report. Corey Stein wrote the first draft of the manuscript. Corey Stein, Luke Devine and Anne McLeod contributed to the critical review of the literature, editing and revising of the manuscript and approved the final version submitted for publication.

Acknowledgements: The authors thank Dr. Graham RocheNagle from the Division of Vascular Surgery at the University Health Network for his assistance in preparing the relevant figures and Dr. Paul Bunce from the Department of Medicine at the University Health Network for advice during the preparation of the manuscript.

The section Cases presents brief case reports that convey clear, practical lessons. Preference is given to common presentations of important rare conditions, and important unusual presentations of common problems. Articles start with a case presentation (500 words maximum), and a discussion of the underlying condition follows (1000 words maximum). Visual elements (e.g., tables of the differential diagnosis, clinical features or diagnostic approach) are encouraged. Written consent from patients for publication of their story is a necessity and should accompany submissions. See information for authors at www.cmaj.ca. 